\title{
Koenen, Erik (Hrsg.) (2016): Die Entdeckung der Kommunikationswissenschaft. 100 Jahre kommunikations- wissenschaftliche Fachtradition in Leipzig: Von der Zeitungskunde zur Kommunikations- und Medienwissenschaft. Köln: Herbert von Halem
}

\author{
Mike Meißner, Universität Freiburg (CH), DCM - Departement für Kommunikationswissenschaft \\ und Medienforschung \\ mike.meissner@unifr.ch
}

Der von Erik Koenen herausgegebene Band, der im Kontext des hundertjährigen Jubiläums des Leipziger Instituts für Kommunikations- und Medienwissenschaft (IfKMW) entstanden ist, zeichnet auf einer ersten Ebene die organisatorische Entwicklung des Instituts nach. Auf einer zweiten Ebene wird die Bedeutung des Leipziger Standortes für die Institutionalisierung der Kommunikationswissenschaft in Deutschland und im deutschsprachigen Raum hervorgehoben. Die acht Beiträge, die klassisch chronologisch angeordnet sind, werden von gleich zwei Vorworten (Sonja Ganguin/Hans-Jörg Stiehler, Oliver Quiring), einer Einführung sowie einer Instituts-Chronik gerahmt. Der eilige Leser ist gut beraten, den Beitrag «Überblick und Einführung» von Erik Koenen zu lesen, in dem die Institutsgeschichte knapp dargestellt wird und die Erkenntnisse der anderen Beiträge jeweils sehr gut eingebettet sind.

Allen anderen, die mehr zu Einzelaspekten wissen wollen, seien die anderen Beiträge empfohlen. Der Vorgeschichte und Gründungsphase des damaligen Instituts für Zeitungskunde, mit denen der Name Karl Büchers eng verbunden ist, sind gleich zwei Beiträge gewidmet. Thomas Wiedemann und Michael Meyen zeichnen die institutionelle Gründungsleistung Büchers nach und versuchen thesenhaft dessen Einfluss «auf die Entwicklung des Fachs Kommunikationswissenschaft in ganz Europa» (S. 60) zu belegen. Sie zeigen, dass es bei den vier von ihnen ausgewählten Dimensionen (Akteure, soziale Strukturen, Forschungsagenda und Lehr- programm, akademischer Reputationsgewinn) Parallelen in der Entwicklung gab. So kamen bspw. viele frühe Fachvertreter aus der journalistischen Praxis, in der Schweiz z. B. Oscar Wettstein, Michael Bühler, Karl Weber und Siegfried Frey (laut Wiedemann/Meyen «ein gebürtiger [!] Journalist», S. 63). Jedoch wird kaum deutlich, inwiefern dies dem Einfluss von Bücher zuzuschreiben ist. Deshalb ist es bedauerlich, dass die beiden Verfasser «ein ausführliches Fazit [für] überflüssig» (S. 73) halten.

Am Beispiel von Büchers Studienprogramm für eine akademische Journalistenausbildung spürt Arnulf Kutsch dagegen Einflüssen auf Bücher nach. Neben dessen grundsätzlichen Standpunkten, die unter anderem von seinem Förderer Albert Schäffle geprägt waren, konzentriert sich der Beitrag auf die Einflüsse aus den USA und Europa, speziell der Schweiz, auf Büchers Programm. Es wird deutlich, dass es erhebliche Überschneidungen mit dem bereits zuvor von Oscar Wettstein entwickelten Zürcher Modell gab. Dies betraf neben dem zweiteiligen theoretischen Studium (sach- und fachwissenschaftliche Veranstaltungen) auch die Ablehnung eines speziellen Examens für Journalisten (vgl. S. 99-103). Dagegen erweiterte Bücher sein Curriculum um einen praktischen Teil, der bereits in weiten Teilen in einem Aufsatz des an der University of Chicago lehrenden George Edgar Vincent skizziert war. Bücher ging bei seiner Auswahl eklektisch vor, was vor allem daran lag, dass er von den verschiedenen Konzeptionen durch persönliche Kontakte, etwa den 
Schweizer Theodor Curti und eine Reihe akademischer Schüler, Kenntnis erlangte. Kutsch konstatiert anhand einiger Beispiele, dass Bücher nicht umfassend über die aktuelle Situation der Journalistenausbildung informiert gewesen ist.

Büchers Nachfolger als Institutsleiter, Erich Everth, der zugleich erster ordentlicher Professor für Zeitungskunde in Leipzig war, widmet sich Erik Koenen im folgenden Beitrag. Es ist ein grosser Verdienst Koenens, Biographie und wissenschaftliche Leistung des lange in Vergessenheit geratenen Everths aufgearbeitet zu haben (vgl. Koenen 2015). Besonders erfreulich ist, dass diese Lücke rechtzeitig zum Institutsjubiläum geschlossen werden konnte.

Die hauptsächlich durch die NS-Studentenschaft erzwungene Ablösung Everths 1933 nach der Machtübergabe an die Nationalsozialisten stellte den Beginn der «Politische[n] Überformung» (Jochen Jedraszczyk) des Leipziger Instituts dar, das ab 1935 Institut für Zeitungswissenschaft hiess. Jedraszczyk beleuchtet kenntnisreich die Zeit von 1933 bis zum Kriegsende, in der das Institut von Hans Amandus Münster geleitet wurde, und verdeutlicht an konkreten Beispielen, wie sich die Institutsmitglieder ganz praktisch in den Dienst der neuen Machthaber stellten. Eine Dissertation über jüdische Journalistinnen und Journalisten in der Weimarer Republik, in der mehrere hundert Menschen namentlich aufgelistet wurden, war bspw. bei diversen Dienststellen von Partei und Staat «als «wertvolles Nachschlagewerk sehr nachgefragt» (S. 175), wie Münster in seinem Gutachten hervorhob.

Nach dem Ende des NS-Regimes erfolgte in Leipzig in sehr kurzer Zeit, bis 1951, zunächst eine Entideologisierung und nach kurzer Rekonstruktion eine Reideologisierung des Fachs. Diesen Prozess mit verschiedenen personellen und institutionellen Veränderungen - zwischenzeitlich gab es gar zwei Institute, die aber bald zusammengelegt wurden - stellt Jedraszczyk mit grosser Detailtreue dar. Besonders interessant ist in diesem $\mathrm{Zu}$ sammenhang die Auflistung der von Gerhard Menz von 1948 bis 1951 betreuten Dissertationen und deren Einordnung durch den Verfasser. Jedraszczyk weist auf funktional-prozessorientierte Ansätze hin, für die Menz gestanden habe. Im Lutherjahr 2017 darf der Hinweis nicht fehlen, dass auch eine Arbeit über den Reformator angefertigt wurde. Der Beitrag schliesst mit einem kurzen Ausblick auf die weitere Entwicklung in der DDR (ab 1954: Fakultät für Journalistik; ab 1969 Sektion Journalistik) und einem Foto des Institutsgebäudes, des «Roten Klosters», wie die Einrichtung später genannt wurde.

Mit den Leipziger Journalistik-Professoren (und einer Professorin) in der DDR beschäftigen sich wiederum Meyen und Wiedemann in Form einer Kollektivbiographie und stossen damit in eine Lücke der fachhistorischen Forschung. Sie liefern u. a. auf der Basis von fünf Zeitzeugeninterviews mit den letzten noch lebenden und sich gesundheitlich dazu in der Lage befindenden Journalistik-Professoren einen Startpunkt für die weitere Erforschung der Journalistenausbildung in der ehemaligen DDR.

Dem letzten zeitlichen Abschnitt, der Zeit seit der Neugründung 1993 als IfKMW bis heute, widmet sich Meyen. Er nutzt dies für eine kritische Bestandsaufnahme des damals geschaffenen Fünf-Säulen-Modells, welches Kommunikations- und Medienwissenschaft sowie Journalistik, PR und Medienpädagogik verbindet und damals wie heute quer zur sonstigen organisatorischen Entwicklung der Fachinstitute im deutschsprachigen Raum steht. Ohne zu einer Apologie ansetzen $\mathrm{zu}$ wollen, ${ }^{1}$ scheint die Aussage gewagt, dass es eine generelle «Tendenz zur Abschottung» (S. 270) zwischen den verschiedenen Abteilungen gegeben hat. Denn sowohl auf der Ebene der Lehre im gemeinsamen Magister- und später auch im Bachelor- und Masterstudiengang KMW als auch in der Forschung gab es durchaus Kooperationen, die teilweise auch in Publikationen mündeten (vgl. Keiderling et al. 2007; Schorb/Stiehler 1996; Steinmetz et al. 2007; Steinmetz/Stiehler 1997). Ferner hätte es zur Transparenz bei-

1 Der Rezensent studierte von 2006 bis 2012 am IfKMW und schloss mit einer Arbeit bei Arnulf Kutsch ab. 
getragen, wenn an geeigneter Stelle darauf hingewiesen worden wäre, dass es sich bei Meyen selbst um einen 'Leipziger handelt, der dort sowohl promoviert als auch habilitiert wurde (bei Arnulf Kutsch).

Insgesamt besticht der Band durch seinen Aufbau: Die Beiträge lassen sich durch gut gestaltete Übergänge auch flüssig am Stück lesen, was für einen Sammelband eher eine Ausnahme ist. Abgesehen von der chronologischen Gliederung, hängt das mit den Verweisen zwischen den einzelnen Texten zusammen, ohne störende Wiederholungen zu produzieren. Zusätzlich sind die Beiträge durch Porträts der tonangebenden Personen, weitere Abbildungen und Tabellen aufgelockert. Die biographischen Skizzen sind kompakt, nur jene zu Hans A. Münster ist auf zwei Beiträge verteilt. Konkrete Angaben zu seinen Aktivitäten nach 1945 finden sich, chronologisch korrekt, in dem Beitrag zu den Jahren nach 1945 (S. 188-189), der sich aber eigentlich nicht mehr mit Münster beschäftigt.

Mit «Die Entdeckung der Kommunikationswissenschaft» liegt eine konzise Darstellung der v. a. organisatorisch-personellen Entwicklung des heutigen Leipziger Instituts vor. Sie sollte nicht nur in der grundständigen Lehre des IfKMW, etwa im Rahmen einer Einführungsvorlesung, Verwendung finden, sondern auch als Anstoss für weitere (fach-)historische Arbeiten innerhalb der deutschsprachigen Kommunikations- und Medienwissenschaft dienen.

\section{Literatur}

Keiderling, Thomas/Kutsch, Arnulf/Steinmetz, Rüdiger (Hrsg.) (2007): Buch - Markt Theorie. Kommunikations- und medienwissenschaftliche Perspektiven. Erlangen: Filos.

Koenen, Erik (2015): Erich Everth - Wissenstransformationen zwischen journalistischer Praxis und Zeitungskunde. Biographische und fachhistorische Untersuchungen. Diss. Universität Leipzig.

Schorb, Bernd/Stiehler, Hans-Jörg (Hrsg.) (1996): Medienlust - Medienlast. Was bringt die Rezipientenforschung den Rezipienten? München: KoPäd-Verlag.

Steinmetz, Rüdiger/Stiehler, Hans-Jörg/Wank, Johanna (2007): Das Leipziger Dokfilm-Festival und sein Publikum II. Eine Nachfolge-Studie 2006 zu Image, Akzeptanz und Resonanz. Leipzig: Leipziger Universitätsverlag.

Steinmetz, Rüdiger/Stiehler, Hans-Jörg (1997): Das Leipziger Dokfilm-Festival und sein Publikum. Eine Studie zu Image, Akzeptanz und Resonanz 1993-1996. Leipzig: Leipziger Universitätsverlag. 